\title{
Hacia un nuevo concepto de la
}

\section{inspección, vigilancia y control \\ del derecho a la salud y del Sistema}

General de Seguridad Social en Salud en Colombia

\section{Towards a new concept of monitoring, control and surveillance of health right and the general social security system for health in Colombia}

\section{$\{\text { Jaime León Gañán Echavarría }\}^{*}$}

Docente Investigador, Universidad de Antioquia. Especialista en Derecho del Trabajo y de la Seguridad Social, Universidad Pontificia Bolivariana. Doctor en Derecho, Universidad Externado de Colombia. Posdoctor, Centro de Estudios Avanzados, Universidad Nacional de Córdoba. Correo electrónico: gananr@une.net.co / DOI: https://doi.org/ro.1860I/25390406.n I.05

Recibido: I de junio de 2016

Aprobado: 23 de agosto de 2016 


\section{RESUMEN}

Con la entrada en vigencia de la Constitución Política de i99i, Colombia pasó a ser un Estado social de derecho; hecho que implicó plantear nuevas formas de concebir, fundamentar y garantizar los derechos. De ahí que tanto derechos como deberes constitucionales y legales se replantearan bajo nuevas concepciones de carácter social, político, económico y jurídico. En este punto, la interpretación de los derechos tuvo que trascender las nociones tradicionales, basadas principalmente en fuentes de derecho de tipo legal, para avanzar hacia un derecho fundamentado en un nuevo sistema de fuentes de derecho, en favor de la persona, la materialización de la dignidad humana y la prevalencia de los derechos inalienables. En este contexto, el derecho a la salud debe ser reinterpretado a la luz del "Bloque de Constitucionalidad" como un derecho humano fundamental, inescindible de otros derechos y deberes. Asimismo, la noción de inspección, vigilancia y control debe extenderse al propio derecho, más allá de su dimensión prestacional y reorientarse hacia su materialización integral, en pro del acceso efectivo y del goce con calidad del derecho fundamental a la salud.

\section{PALABRAS CLAVE}

Derecho fundamental a la salud; inspección, vigilancia y control (IVc); sistema general de seguridad social en salud (sGSss)

\section{ABSTRACT}

As the Political Constitution of r99 I entered into effect, Colombian State adhered to a social rule of Law. That obliged the Colombian State to consider new ways to conceive, underlie and guarantee citizens' rights. Hence both constitutional and legal rights and duties were redefined in light of new social, political, economic and legal concepts. Thus, the interpretation of rights went beyond traditional notions based primarily on legal rate sources, to a system based on new sources that favor individuals, human dignity and the prevalence of inalienable rights. For this reason, the right to health must be reconsidered considering "the body of Constitutional law" as a fundamental right that is inseparable from other rights and duties. Furthermore, the notion of monitoring, control and surveillance should be extended to law far beyond its social benefit dimension, and be realigned towards its comprehensive materialization; in order to ensure the effective access and the enjoyment of the right to health.

\section{KEYWORDS}

Fundamental right to health; General Social Security System for Health (GSSSH); monitoring, control and surveillance (MCs) 


\section{INTRODUCCIÓN}

Como principal desarrollo legislativo de los artículos 48 y 49 de la Constitución Política de I991, entre otros relacionados con los derechos a la Seguridad Social y a la Salud, respectivamente, se creó, con la Ley ıoo de I993, el denominado Sistema de Seguridad Social Integral $(\mathrm{sssi})^{\mathrm{I}}$ en Colombia. Este Sistema está compuesto por tres subsistemas denominados, en su orden: Sistema General de Pensiones (sGP), Sistema General de Seguridad Social en Salud (sgsss) y Sistema General de Riesgos Profesionales - hoy denominado Sistema General de Riesgos Laborales (SGRL) según la Ley I 562 de 2012-. La legislación también creó un conjunto de programas adicionales a los subsistemas citados, bajo la denominación de Servicios Sociales Complementarios (ssc).

El sGsss ${ }^{2}$ contempló la existencia simultánea de dos regímenes: el régimen contributivo y el régimen subsidiado en salud. El sGsss tiene como objetivos: regular el servicio público de salud, crear condiciones para el acceso de toda la población al servicio de salud en todos los niveles de atención, cubrir las contingencias de enfermedad general y maternidad de sus afiliados y beneficiarios $y$, como corolario, garantizar a todas las personas el acceso a los servicios de promoción, protección y recuperación de la salud.

Con tal fin, la citada Ley roo de I993 estipuló la implementación de un plan obligatorio de salud ( $(\mathrm{POS})^{3}$, como un conjunto básico de servicios en salud para los afiliados y beneficiarios de dicho Sistema. El pos pretende, en consecuencia, cubrir las necesidades en salud y satisfacer el derecho a la salud de los afiliados y beneficiarios del Sistema, por medio de la implementación, reconocimiento y garantía de servicios de promoción de la salud, prevención de la enfermedad, medicamentos, intervenciones, hospitalización y rehabilitación, entre otros servicios y contenidos ${ }^{4}$.

Según el artículo 49 de la Constitución Política, la propia Ley roo de I993, las leyes I I 22 de 2007 y I438 de 20 I I, por las cuales se ha reformado el SGSSs; y la actual Ley I 75 I de 2015 son coincidentes en determinar que el Derecho Fundamental a la Salud y los servicios derivados del mismo, en su dimensión o faceta prestacional como servicios públicos esenciales, deben ser desarrollados con eficiencia, oportunidad, accesibilidad, disponibilidad, aceptabilidad, integralidad, universalidad, calidad y lógicamente con calidez, entre otros principios rectores que buscan el acceso real y material de todas las personas con equidad y

I Ley Ioo de I993, sobre el sistema de seguridad social integral, art. $\mathrm{I}^{\mathrm{o}} \mathrm{y}$ art. $5^{\circ}$.

2 Ibíd., Libro II, arts. I 52-248.

3 Ibíd., art. I62; y Ley I438 de 20 I I, sobre la reforma del sistema general de seguridad social en salud, art. 25

4 Ley I 75 I de 20 I 5, sobre el derecho fundamental a la salud, art. I 5. 
progresividad al goce de tal derecho5. En ese contexto la Inspección, Vigilancia y Control (IVc) del derecho a la salud y del propio sGsss se constituyen en elementos básicos de fortalecimiento de los elementos esenciales que materializan la prestación oportuna y con calidad de los servicios de salud.

En voces de algunos actores del sGsss, la tarea de la IVC no es tan eficiente como se desearía, debido a múltiples factores que van desde el propio diseño de tal sistema, hasta algunas condiciones de tipo logístico. Por ello, este artículo analiza tal situación con el fin de proponer algunas alternativas de solución de política pública o de tipo normativo en relación a la IVc y establecer si es necesaria su reorientación hacia un real Sistema de Inspección, Vigilancia y Control en pro del goce efectivo y con calidad del Derecho Fundamental a la Salud, y de su dimensión prestacional como servicio público esencial.

\section{Hacia un NUEVo CONCEPTO Y CARActerización DE LA INSPECCIÓN, VIGILANCIA Y CONTROL}

En el devenir histórico, social, político, jurídico del concepto de salud y del propio concepto del derecho a la salud es posible afirmar que Colombia ha transitado por diferentes etapas de estructuración de tales nociones. Algunas veces, en franca proyección hacia una concepción integral e integradora y en otras, en franco retroceso hacia concepciones más limitadas y restringidas de salud y de derecho a la salud.

En los últimos veinte años podría decirse que en Colombia la salud transitó una delgada línea, desde concepciones meramente de tipo biologista, hasta concepciones de tipo integral. Se evolucionó, por lo menos formalmente, desde la concepción de la salud como un problema de tipo en esencia físico a una dimensión de tipo holístico, en la cual la salud es multidimensional y, por tanto, se integra de aspectos físicos, pero también de contenido mental, psicológico, social, familiar, espiritual, entre otros ${ }^{6}$.

De igual forma, el concepto de salud trasmuta, por lo menos en teoría, desde la concepción de la ausencia de enfermedad como parámetro básico de salud, hasta la concepción de salud desde la promoción, la prevención y como el

5 Cfr. "Observaciones generales aprobadas por el Comité de Derechos Económicos, Sociales y Culturales, Observación General n. ${ }^{\circ}$ I4”, Comité del Pacto de Derechos Económicos, Sociales y Culturales, consultado en septiembre de 2016

6 Sentencia T-or6 de 2007, M.P. Humberto Antonio Sierra Porto; y Sentencia T-760 de 2008, M.P. Manuel José Cepeda Espinosa. 
mayor nivel posible de bienestar y calidad de vida ${ }^{7}$, o en términos de la comunidades indígenas como "buen vivir".

En tal contexto de evolución general del concepto de salud, también fue cambiando el concepto del derecho a la salud y lógicamente de su naturaleza jurídica. De no estar positivizado en la Constitución de I986, lo encontramos en forma expresa en el artículo 49 de nuestra Constitución de i99 I y en otros, tales como los artículos: 42, 44, 50 y 53. Por su ubicación en el artículo 49, que corresponde a los denominados por nuestra Constitución, como derechos económicos, sociales y culturales, por mucho tiempo se entendió como un típico derecho asistencial o programático, siendo la Corte Constitucional, a través de su múltiple jurisprudencia acerca de la naturaleza jurídica, la Corporación que fue consolidando la esencia del derecho a la salud como un derecho fundamental y autónomo, alejándolo de la concepción de un mero servicio público ${ }^{9}$. Lo anterior, en articulación del llamado "Bloque de Constitucionalidad" y de su aplicación con respecto a un concepto ampliado del derecho a la salud y de sus elementos esenciales.

En ese orden de ideas, el derecho a la salud evoluciona de ser un mero servicio público esencial revestido de alguna fundamentalidad para efectos de su tutela, a un verdadero derecho fundamental autónomo en lo individual y en lo colectivo, tal como hoy lo refiere la Ley I75 I de 2015 o "Ley Estatutaria en Salud" " , con una dimensión prestacional, como servicio público esencial.

En este punto histórico, jurídico, social y político del derecho a la salud es que la Ivc debe, igualmente, evolucionar hacia nuevas concepciones, características y formas de materializarse. Tradicionalmente se ha entendido el tema de la IVc como un concepto ligado en esencia a los servicios públicos, en especial aquellos considerados como de tipo esencial. Por ello, la ivc de la salud en Colombia ha estado ligada al seguimiento y supervisión de los servicios de salud procurados por los diferentes actores del sGsss y así lo han contemplado la Ley IoO de I $993^{\text {II }} \mathrm{y}$, en concreto, las leyes I I 22 de 2007 y I 438 de 20 I r. No obstante, podríamos afirmar que en la actualidad la IVC trasciende el concepto cerrado del sGSss y debe transmutar, igual que el concepto de salud y de derecho a la salud, hacia consideraciones más amplias. En particular, a la concepción de la IVc del acceso efectivo, del cumplimiento y del goce real del Derecho Fundamental a la

Sentencia C-252 de 20 го, M.P. Jorge Iván Palacio Palacio; Sentencia C-79 I de 20 I r, M.P. Humberto Antonio Sierra Porto; y Sentencia C-3 I3 de 20I4, M.P. Gabriel Eduardo Mendoza Martelo.

Cfr. Proyecto de Ley, sobre el Sistema Indígena de Salud Propia e Intercultural-sisPi.

Jaime León Gañán Echavarría, "De la Naturaleza Jurídica del Derecho a la Salud en Colombia”, $R e$ vista Monitor Estratégico, núm. 3 (enero-junio de 2013): 7-19.

Cfr. Sentencia C-3 13 de 2014.

Ley roo de I 993, Libro II, art. I 52-248; Ley I 22 de 2007, sobre modificaciones en el Sistema General de Seguridad Social en Salud; y Ley I 438 de 20 I I. 
Salud ${ }^{\mathrm{I} 2}$, y lógicamente también, a la ivc de su dimensión como servicio público esencial.

Desde la concepción de la ivc del derecho como tal, habría que procurar su garantía como derecho integral e integrador ${ }^{13}$ : integral, porque refiere a dimensiones holísticas e integrador, en tanto que se encuentra en articulación necesaria con otros derechos, deberes y libertades. En tal sentido, la Ivc debe informar sobre el cumplimiento, respeto y protección que los Estados le deben a tales derechos y a su materialización universal y equitativa; y en esa misma vía, a los determinantes estructurales y sociales que llenan de igualdad material o fáctica tales derechos. Igualmente, se estaría supervisando que las características propias de un derecho fundamental se materialicen, esto es que en la realidad sea imprescriptible, universal, inherente a cada persona, sistémico, vinculante, inalienable, irrenunciable, entre otros aspectos del mismo. A la vez, que se cumplan con las garantías constitucionales de su protección, entre ellas: acción especial para su protección, en nuestro caso vía acción de tutela ${ }^{\mathrm{I}}$; que no pueda ser limitado en estados de excepción ${ }^{15}$; que se desarrolle a través de ley estatutaria ${ }^{16}$; que sea de aplicación inmediata, y que su contenido se determine a la luz del artículo 377 de la Constitución de r99i.

Desde la dimensión prestacional del derecho a la salud como un servicio público esencial, entonces, la Ivc también debe procurar porque la prestación del mismo, ya sea por particulares o por agentes públicos o mixtos, cumpla con los principios jurídicos propios de los mismos, con los caracteres jurídicos que los informan y con los elementos esenciales de los servicios públicos de salud ${ }^{17}$. Además de los principios de beneficencia, de no maleficencia, de benevolencia, de veracidad, de confidencialidad de la información que deben cumplir los profesionales de la salud.

A la luz no solo de las sentencias que han considerado la salud como un verdadero derecho fundamental autónomo, sino también bajo los actuales parámetros de la Ley I 75 I de 20 I 5 .

Jaime León Gañán Echavarría, Los muertos de Ley ioo. Prevalencia de la libertad económica sobre el derecho fundamental a la salud: una razón de su ineficacia. Caso del Plan Obligatorio de Salud del Régimen Contributivo. (Medellín: Imprenta Universidad de Antioquia, 2013)

Constitución Política de Colombia, r99 r, art. 86.

Ibíd., art. 93; Ibid. art. 2 I4, num. 2; Ley I 37 de I994, sobre los estados de excepción en Colombia, art. 4. ${ }^{\circ}$ y Convención Americana de Derechos Humanos, art. 27.

C.P. art. $5^{2}$.

También se debe enfatizar como complemento a los elementos esenciales enunciados, los conceptos determinados por la Observación General 3, aprobada por el Comité de Derechos Económicos, Sociales y Culturales aplicable a todos los compromisos y derechos estipulados en el Pacto, de los cuales se resaltan: la no discriminación, la equidad, la progresividad, la irreversibilidad, la gratuidad y la participación. Los elementos esenciales y las características comunes del derecho al trabajo y del derecho a la salud referidos pretenden, sin duda, darle cumplimiento pleno y efectivo a tales derechos. Cfr. "Observaciones generales aprobadas por el Comité de Derechos Económicos, Sociales y Culturales, Observación General n. ${ }^{3}$ 3, Comité del Pacto de Derechos Económicos, Sociales y Culturales, consultado en septiembre de 2016 , https://goo.gl/odtKPe. 
Entre los principios jurídicos de los servicios públicos estarían: la accesibilidad, la educación, la competencia, la proporcionalidad, la vigencia técnica, la regulación, la fiscalización y protección ${ }^{18}$. Dentro de los caracteres jurídicos estarían: la continuidad, la regularidad, la uniformidad, la generalidad, la obligatoriedad, la calidad y la eficiencia ${ }^{19}$. Finalmente, en el marco de los elementos esenciales relacionados directamente con la salud se tendrían: la accesibilidad, la disponibilidad, la aceptabilidad, la calidad ${ }^{20}$ y la calidez.

En consecuencia de las premisas anteriores, el Sistema de Inspección, Vigilancia y Control del sGsss creado por la Ley I 22 de 2007 debería expandirse hacia una concepción más amplia y, por ende, partir de indicadores generales del cumplimiento del derecho fundamental a la salud y en forma concomitante realizar el seguimiento, control y mejoramiento a los específicos indicadores del sGSSs como tal.

En conclusión, con la entrada en vigencia de la Ley I $_{75}$ I de 2015, que formaliza el reconocimiento del Derecho a la Salud como un verdadero derecho fundamental y autónomo en lo individual y en lo público, la Ivc debe superar las barreras del propio Sistema y verificar las condiciones de materialización real y efectiva, si se quiere progresiva y no reversible, del derecho a la salud y de su propia noción ${ }^{21}$. Para ello se tendrían que fortalecer las condiciones estructurales y sociales con el fin de que el derecho como tal se efectivice. Lo anterior podría

Roberto Dromi. Derecho Administrativo, ro. ${ }^{\mathrm{a}}$ ed. (Buenos Aires: Ciudad Argentina, 2004) Ibíd.

Es de anotar que el comité de Derechos Económicos, Sociales y Culturales se refiere al derecho a la salud como: “...el derecho de toda persona al disfrute del más alto nivel posible de salud física y mental..." Precisamente mediante la Observación General i4 se amplía la concepción del derecho a la salud al definirse como un "... derecho humano fundamental e indispensable para el ejercicio de los demás derechos humanos..." y al determinarse que "El derecho a la salud está estrechamente vinculado con el ejercicio de otros derechos humanos y depende de esos derechos..." Según la misma observación, tal derecho no debe entenderse sólo como un derecho a estar sano, entraña también libertades y derechos. Entre las libertades está el derecho a controlar la salud y el propio cuerpo, con la inclusión de la libertad sexual y genésica. El Comité interpretó, además, que el derecho a la salud es un derecho inclusivo en cuanto no sólo abarca la atención en salud oportuna y apropiada sino también sus principales factores determinantes. Cfr. "Observaciones generales aprobadas por el Comité de Derechos Económicos, Sociales y Culturales, Observación General N. ${ }^{\circ}$ I 4", Comité del Pacto de Derechos Económicos, Sociales y Culturales, consultado en septiembre de 20I6, https://goo.gl/odtKPe

También es de anotar que el Protocolo Adicional a la Convención Americana sobre Derechos Humanos en materia de derechos económicos, sociales y culturales, estipula que toda persona tiene derecho a la salud, entendida “... como el disfrute del más alto nivel de bienestar físico, mental y social”. Así mismo que se reconoce este derecho como un "... bien público". Cfr. ozA, "Protocolo adicional a la Convención Americana sobre Derechos Humanos en materia de derechos económicos, sociales y culturales 'protocolo de san salvador", Departamento de Derecho Internacional, Organización de los Estados Americanos (oEA), consultado en septiembre de 20I6, https://goo.gl/vTjlya. De acuerdo con la Constitución de la Organización Mundial de la Salud (oмs): “... La salud es un estado de completo bienestar físico, mental y social, y no solamente la ausencia de afecciones o enfermedades". Cfr oms, "Constitución de la Organización Mundial de la Salud" (Documento básico, suplemento de la $45 .{ }^{\text {a }}$ ed.), https://goo.gl/L4ysdR 
hacerse desde las políticas públicas para la salud; los planes de desarrollo basados en la concepción del derecho fundamental y no solo en la de servicios de salud; y los planes o programas de verificación sistemática de los indicadores de la materialización y del goce efectivo del derecho a la salud en su dimensión fundamental, como en su dimensión de servicio público esencial previa la concertación de metas en los planes antes mencionados y, que cubran tanto, la salud pública como la individual en todas sus fases de promoción, prevención, diagnóstico, atención, hospitalización, medicamentos, rehabilitación, etc.

La pregunta que podría surgir en relación de lo arriba referido, y de una nueva concepción de la IVc integral, podría ser si existe efectivamente un sistema de ivc que permita tal función desde el derecho y desde el servicio público esencial en forma oportuna, eficiente, proactiva, dinámica, colaborativa, adecuada y propositiva.

\section{Del estado actual de cosas en la inspección, VIGILANCIA Y CONTROL EN SALUD}

En la actualidad, y según el artículo 36 de la Ley i 22 de 2007, se encuentra creado el Sistema de Inspección, Vigilancia y Control del sGsss como un conjunto de normas, agentes y procesos articulados entre sí, en cabeza de la Superintendencia Nacional de Salud ${ }^{22}$, de acuerdo con sus competencias constitucionales, legales y sin perjuicio de las facultades asignadas al Instituto Nacional de Salud y al Invima.

Según la Ley i 22 de 2007, los ejes del sistema de IVc de la Superintendencia Nacional de Salud para ejercer sus funciones serán: financiamiento, aseguramiento, prestación de servicios de atención en salud pública, atención al usuario y participación social, eje de acciones y medidas especiales, información y focalización de los subsidios en salud ${ }^{23}$.

De igual forma, según el artículo 35 de la Ley I 22 de 2007, se adoptan las siguientes definiciones para la Ivc:

- Inspección: La inspección, es el conjunto de actividades y acciones encaminadas al seguimiento, monitoreo y evaluación del Sistema General de Seguridad Social en Salud y que sirven para solicitar, confirmar y analizar de manera puntual la información que se requiera sobre la situación de los servicios de salud y sus recursos, sobre la situación jurídica, financiera, técnica-científica, administrativa y económica de las entidades sometidas a vigilancia de la Superintendencia Nacional de Salud dentro del ámbito de su competencia... Son funciones de inspección entre otras las visitas, la

22 Ley I 22 de 2007 , art. 39.

23 Ibíd., art.37. 
revisión de documentos, el seguimiento de peticiones de interés general o particular y la práctica de investigaciones administrativas.

- Vigilancia: La vigilancia, consiste en la atribución de la Superintendencia Nacional de Salud para advertir, prevenir, orientar, asistir y propender por que las entidades encargadas del financiamiento, aseguramiento, prestación del servicio de salud, atención al usuario, participación social y demás sujetos de vigilancia de la Superintendencia Nacional de Salud cumplan con las normas que regulan el Sistema General de Seguridad Social en Salud para el desarrollo de este.

- Control: El control consiste en la atribución de la Superintendencia Nacional de Salud para ordenar los correctivos tendientes a la superación de la situación crítica o irregular (jurídica, financiera, económica, técnica, científico-administrativa) de cualquiera de sus vigilados y sancionar las actuaciones que se aparten del ordenamiento legal bien sea por acción o por omisión.

Según el artículo I 2 I de la Ley I 438 de 20 I I, tales funciones ${ }^{24}$ se ejercerán por la Supersalud sobre un universo comprendido por:

Las Entidades Promotoras de Salud del Régimen Contributivo y Subsidiado, las Empresas Solidarias, las Asociaciones Mutuales en sus actividades de Salud, las Cajas de Compensación Familiar en sus actividades de salud, las actividades de salud que realizan las aseguradoras, las Entidades que administren planes adicionales de salud, las entidades obligadas a compensar, las entidades adaptadas de Salud, las administradoras de riesgos profesionales en sus actividades de salud. Las entidades pertenecientes al régimen de excepción de salud y las universidades en sus actividades de salud, sin perjuicio de las competencias de la Superintendencia de Subsidio Familiar... ${ }^{25}$. Las Direcciones Territoriales de Salud ${ }^{26}$ en el ejercicio de las funciones que las mismas desarrollan en el ámbito del sector salud, tales como el aseguramiento, la inspección, vigilancia y control, la prestación de servicios de salud y demás relacionadas con el sector salud... Los prestadores de servicios de salud públicos, privados o mixtos... ${ }^{27}$. La Comisión de Regulación en Salud ${ }^{28}$ y el Fondo de Solidaridad y Garantía, Fosyga, o quienes hagan sus veces... Los que exploten, produzcan, administren u operen, bajo cualquier modalidad, el monopolio rentístico de loterías, apuestas permanentes y demás modalidades

Ley I 438 de 20 I I, art. i 8 
de los juegos de suerte y azar... Los que programen, gestionen, recauden, distribuyan, administren, transfieran o asignen los recursos públicos y demás arbitrios rentísticos del [sGsss]... Las rentas que produzcan cervezas, sifones, refajos, vinos, aperitivos y similares y quienes importen licores, vinos, aperitivos y similares y cervezas... Los que exploten, administren u operen, bajo cualquier modalidad, el monopolio rentístico de los licores ${ }^{29}$.

De un breve análisis se colige que el universo de actores vigilados por la Supersalud es de una gran magnitud. Se estima que pueden ser más de i 2 ooo los sujetos que se deben vigilar en todo el territorio nacional $3^{\circ}$. Si a ello se le agrega que la planta de personal de la entidad no supera las 700 personas y que la entidad aún se encuentra concentrada en la capital, entonces sería muy entendible que no fuera posible una IVc georreferenciada, oportuna, completa y eficaz, pese a los grandes esfuerzos que se perciben por parte de la dirección de tal Entidad, encaminados a mejorar sus procesos y acercarse a la comunidad ${ }^{3 \mathrm{I}}$.

De otra parte, son múltiples los actores que también tienen facultades de inspección, vigilancia o control sobre el sGsss, en todo o en partes determinadas, y que a su vez son sujetos de vigilancia de la Superintendencia Nacional de Salud. Entre ellos, podemos encontrar: al propio Ministerio de Salud y Protección Social ${ }^{22}$, al Instituto Nacional de Salud ${ }^{33}$, al Invima ${ }^{34}$, a la

29 Según el informe de 2013 de Gestión de la Supersalud son alrededor de r 82 vigilados.

30 Ver informes de Gestión de la Superintendencia, años 201 2-20I3.

3 I Ver la Estrategia de Organización en el Territorio implementada por la Supersalud como forma de acercar la entidad y sus procesos a la comunidad en general.

De acuerdo con el Decreto 4IO7 de 20 I I, entre otras funciones, el Ministerio de Salud y protección Social adelanta los procesos de coordinación con relación a las instituciones prestadoras de servicios de salud que se encuentren adscritas o vinculadas al sGsss y, por ende, frente a las cuales media control de tutela, así como en relación con las demás instituciones prestadoras relacionadas con el sistema. También ejerce las funciones de inspección y dictamen sobre el ejercicio de profesiones y la formación de todo tipo de recurso humano para el sector que adelantan las instituciones públicas, privadas o de cualquier naturaleza, que forman parte del sGsss, de acuerdo con lo establecido en este decreto y en la Ley ıo de I990, sobre la reorganización del Sistema Nacional de Salud. Lógicamente también dirige y orienta el Sistema de Vigilancia en Salud.

33 Cfr. Decreto Ley 4rog de 20 i I, art.3. Se resalta el Sistema Nacional de Vigilancia en Salud Pública (sivigila), que se ha creado para realizar la provisión en forma sistemática y oportuna, de información sobre la dinámica de los eventos que afecten o puedan afectar la salud de la población colombiana, con el fin de: orientar las políticas y la planificación en salud pública, tomar las decisiones para la prevención y control de enfermedades y factores de riesgo en salud, optimizar el seguimiento y evaluación de las intervenciones, racionalizar y optimizar los recursos disponibles y lograr la efectividad de las acciones en esta materia, propendiendo por la protección de la salud individual y colectiva.

34 Entre otras funciones del Invima se encuentra la de diseñar y desarrollar el Sistema Nacional de Vigilancia Sanitaria, ejercer las funciones de inspección, vigilancia y control a los establecimientos productores y comercializadores de los productos a que hace referencia el artículo 245 de la Ley roo de I 993 y en las demás normas que lo modifiquen o adicionen, sin perjuicio de las que en estas materias deban adelantar las entidades territoriales, durante las actividades asociadas con su producción, importación, exportación y disposición para consumo; identificar y evaluar las infracciones a las normas sanitarias y 
$\mathrm{UGPP}^{35}$, a las superintendencias de industria y comercio (SIC) ${ }^{36}$, a la financiera ${ }^{37}$ y a la Supersociedades ${ }^{3}$, a la Contraloría General de la Republica ${ }^{39}$, a la Procuraduría

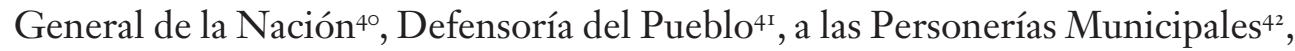

a los procedimientos establecidos, adelantar las investigaciones a que haya lugar y aplicar las medidas sanitarias y las sanciones que sean de su competencia, de conformidad con la Ley 9 de 1979 y demás normas reglamentarias; dirigir y hacer cumplir en todo el país las funciones de control de calidad y vigilancia sanitaria de los productos de su competencia; realizar el control sanitario sobre la publicidad de los productos establecidos en el artículo 245 de la Ley ioo de 1993 y las normas que lo modifiquen o adicionen de conformidad con lo dispuesto en la Ley 9 de 1979 y sus decretos reglamentarios y en las demás normas que se expidan para el efecto; desarrollar el sistema de autorización y verificación internacional para productos objeto de vigilancia, de acuerdo con la normatividad vigente; evaluar y adoptar, en el marco de sus competencias, las medidas que sean necesarias para facilitar los procesos de admisibilidad sanitaria que inicie el país en los mercados internacionales y coordinar con el Instituto Colombiano Agropecuario (ICA) y las demás entidades públicas, las acciones a adelantar.

Además de aportar a la construcción de una sólida cultura de cumplimiento en el pago de los aportes al Sistema de la Protección Social, la Unidad Administrativa Especial de Gestión Pensional y Contribuciones Parafiscales de la Protección Social, tiene entre sus funciones la de solicitar la información relevante que tengan las diferentes entidades, administradoras y órganos vigilancia y control del Sistema de la Protección Social. Así como la de efectuar las labores de coordinación y seguimiento a los procesos de determinación y cobro, con base en la información que le remitan las administradoras, órganos de control y vigilancia y demás entidades del Sistema de la Protección Social. Cfr. Decreto I69 de 2008 y normas concordantes.

36 La sic salvaguarda los derechos de los consumidores, protege la libre y sana competencia, actúa como autoridad nacional de la propiedad industrial y defiende los derechos fundamentales relacionados con la correcta administración de datos personales, Protección al Consumidor. Protección de la competencia. La Superintendencia es responsable de vigilar la observancia de las disposiciones contenidas en el estatuto del consumidor, Ley I480 de 20 I I, en tal virtud tramita las denuncias que se presentan e inicia investigaciones de oficio tendientes a establecer su contravención. En este campo tiene facultades administrativas para ordenar la suspensión de conductas ilegales, sancionatorias para reprimir a los infractores y jurisdiccionales para resolver sobre la garantía mínima presunta. Cfr. "Objetivos y funciones", Superintendencia de Industria y Comercio (sIC), consultado en septiembre de 20I6, https:// goo.gl/hPNFCv; Decreto I663 de I994; y Ley I340 de 2009.

Cfr. Decreto 4185 de 20 I I, sobre la reasignación de funciones entre entidades y organismos del Estado, art. I. ${ }^{\circ}$.

38 Cfr. Ley 222 de I995, sobre modificaciones al Libro II del Código de Comercio y n nuevo régimen de procesos concursales, art.228.

39 Cfr. "Funciones de la Contraloría General de la República", Contraloría General de la República, consultado en septiembre de 2016, https://goo.gl/tEtSpv.

Cfr. "Objetivos y funciones", Procuraduría General de la Nación, consultado en septiembre de 20I6, https://goo.gl/MIoRvJ

Cfr. "Defensorías delegadas. Defensoría Delegada para la Salud, la Seguridad Social y la Discapacidad", Defensoría del Pueblo, consultado en septiembre de 20r6, https://goo.gl/kWD8ko; Resolución núm. 404 del I 7 de junio de 2002; e informes sobre la Acción de Tutela en Salud realizados por la Defensoría.

Se resalta como función el velar por la promoción y protección de los derechos humanos, vigila el debido proceso, la conservación del medio ambiente, el patrimonio público y la prestación eficiente de los servicios públicos, garantizando a la ciudadanía la defensa de sus derechos e intereses. Cfr. Ley I36 de 1994, sobre la modernización, organización y funcionamiento de los municipios, art. I 78 ; y Ley I55 I de 20I2, sobre normas para modernizar la organización y el funcionamiento de los municipios, $\operatorname{art.} 38$ 
a los propios entes territoriales ${ }^{43}$, a la ciudadanía ${ }^{44}$ y a la Rama Judicial ${ }^{45}$, en lo pertinente.

La multiplicidad de actores que, a su vez, tienen facultades de inspección, vigilancia o de control, según el caso, puede ser una fortaleza para consolidar un real sistema de inspección, vigilancia y control. No obstante, también puede ser una debilidad para su fortalecimiento, toda vez que es posible que tales facultades se diluyan, se contrapongan, o que simplemente no se realice una eficiente coordinación de tales potestades. Máxime cuando en algunos actores se puedan dar a la vez varias posiciones encontradas como la de ser vigilante y a la vez proveedor de su vigilado ${ }^{46}$.

De lo anterior podrían derivarse varias preguntas: ¿Cómo se entrecruzan las diferentes potestades o facultades de IVC de los diferentes actores referidos? ¿Quién vigila a quién realmente? ¿Quién vigila a quien vigila? ¿Quién vigila en esencia a los usuarios? ¿Quién vigila a los comerciantes de medicamentos? ¿Quién vigila a las farmacéuticas y droguerías? ¿Quién vigila a los profesionales de la salud? ¿Cómo es el entramado de la Ivc en Colombia?

Quizás habría que desarrollar un artículo por cada pregunta realizada, pero a continuación se hacen breves comentarios de las principales fortalezas y debilidades de la Ivc relacionadas a tales inquietudes. En cuanto a cómo se entrecruzan las potestades de ivc entre los diferentes actores, sería posible analizar una cuestión inicial relacionada con los factores de centralización y descentralización de cada agente ${ }^{47}$. En efecto, una fortaleza podría ser la centralización de tales facultades con relación a un Sistema de Salud que igual pretende la

Son múltiples las competencias de los municipios, departamentos y distritos en el tema de la salud y en especial en los relacionados. Con relación a los Municipios se resalta la ivc con relación al Sistema Obligatorio de Garantía de la Calidad, al aseguramiento Régimen Subsidiado, aseguramiento Régimen Contributivo evasión y elusión de aportes, salud pública. En cuanto a los departamentos se resalta la ivc con relación al Sistema Obligatorio de Garantía de la Calidad en lo de su competencia, lo relacionado con la construcción y mantenimiento de IPS-Centros de Atención al Anciano, Bancos de Sangre, procesos de referencia y contrarreferencia, Centros Regulatorios de Urgencias, entre otras facultades sancionatorias o de control. Cfr. Ley 7I 5 de 200I; Ley I55 I de 20I2; Ley I I 22 de 2007;

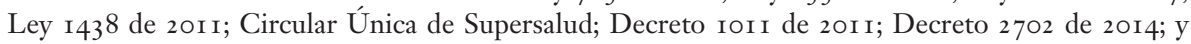
Resolución 2003 de 2014.

A través de veedurías en salud, copagos, ligas de usuarios. Cfr. Ley I34 de I994; y Decreto I 757 de I994.

45 Ver, por ejemplo, la Comisión Especial de Seguimiento a la Sentencia T-760 de 2008, las audiencias celebradas en su desarrollo y los Autos respectivos.

Cfr. Pars, "Diagnóstico del Actual Modelo de Gestión de Inspección, Vigilancia y Control" en Proyecto Evaluación y Reestructuración de los Procesos, Estrategias y Organismos Públicos y Privados Encargados de Adelantar las Funciones de Vigilancia y Control del Sistema de Salud. (Informe final, Bogotá: Ministerio de la Protección Social).

Ramiro Guerrero et al., "La doble descentralización en el sector salud: Evaluación y alternativas de política pública" (Borrador de Informe Final, Concurso de Investigación Fondo Germán Botero de los Ríos, PROESA; Universidad Icesi, octubre de 20I3). 
unificación. No obstante, la prestación de los servicios de salud normalmente son georreferenciados y, por lo tanto, el acercamiento de la Ivc debe ser de tipo territorial o descentralizado. Por lo anterior, la cuestión de quién vigila a quién realmente depende del grado real de capacidad territorial que se tenga para tal labor. En este sentido es probable que la Ivc lejana del servicio real no pueda dar resultados confiables y veraces; y que las medidas de control no sean totalmente oportunas y eficientes.

Aun cuando existen múltiples normativas que referencian las competencias de IVC de los organismos o entidades antes citadas, también parece ser cierto que no es completamente claro quién vigila a los que vigilan. Ello, sin duda, podría ser fuente de multiplicidad de funciones, de vacíos reales en el proceso de IVC y, por supuesto, de conflicto de intereses, como cuando la Supersalud tiene que vigilar al propio Ministerio de Salud y Protección Social, del que depende por adscripción administrativa y funcional; o como cuando las farmacéuticas o droguerías, estando estrechamente ligadas al tema de la salud y del derecho a la salud, no son vigiladas por la Supersalud.

En otra órbita de preguntas, a pesar de existir una norma vigente, nos podríamos cuestionar por quién en esencia y materialmente realiza la IVC a los usuarios y a los profesionales de la salud, sin duda dos actores de gran trascendencia al interior del actual sGsss ¿Existen mecanismos efectivos de IVC para determinar la eficacia o no de las actuaciones de los profesionales de la salud o para determinar las posibles conductas abusivas por parte de algunos usuarios? ¿Existen informes e indicadores de seguimiento a tales conductas?

Tal vez una gran pregunta es si la Ivc realizada desde la vigencia del actual SGSss ha sido eficaz, eficiente y oportuna. De esta pregunta se pueden derivar otras como: ¿La IVc ha arrojado datos confiables? ¿Se han interpuesto sanciones ejemplificantes para los actores que han utilizado el Sistema en su beneficio?, ¿La IVC se ha desarrollado como un verdadero sistema o, por el contrario, ha sido fragmentada, normalmente no oportuna, y posiblemente poco eficiente?

\section{Hacia Un NUEVo Sistema de INSPECción, VIGILANCIA Y CONTROL EN SALUD}

Si acogemos que el derecho a la salud actualmente

“... emerge como un concepto integral e integrador; integral porque cobija al ser humano en todas sus dimensiones, no sólo física sino también mental, emocional, espiritual, psicológica, y porque además lo abarca desde su individualidad, pero también lo hace desde su connotación pública, colectiva, comunitaria y social; e integrador porque se encuentra relacionado en forma inescindible con otros 
derechos, libertades y deberes así como con otros determinantes sociales y estructurales necesarios para su materialización..." ${ }^{48}$;

que la visión integral de la salud se encuentra supeditada a unos claros determinantes de la misma, siendo estos: biológicos, ambientales, servicios de salud y sociales; y que el enfoque de los determinantes ha dado una connotación importante a la visión integral de la salud, en cuanto que la salud de las personas y de las comunidades no solo depende de factores meramente biológicos y de los servicios de salud existentes, sino también, y en forma importante, de las condiciones ambientales, muy especialmente de las condiciones sociales en que viven y laboran las personas y las comunidades ${ }^{49}$. Entonces, el derecho a la salud tendría una connotación más amplia respecto de los modelos tradicionales ${ }^{50} \mathrm{y}$, por ende, sus componentes se ampliarían. En ese contexto, la solidaridad social se redefine en función de la visión integral de la salud, y ella es responsabilidad de todos ${ }^{51}$.

Por otra parte, si atendemos a que la salud se convierte en un tema sistémico, social y colectivo, un tema entendido integralmente no solo como una problemática de servicios tradicionales de salud sino también de salud mental, espiritual, reproductiva, ocupacional, social, tanto desde la perspectiva individual como desde la colectiva y pública ${ }^{52}$ y, en relación directa con otros derechos, deberes y libertades constitucionales; entonces la IVC también debe ser un tema social, colectivo, integral, un tema de Estado ${ }^{53}$ y no solo de Gobierno; un tema estructural y no solo coyuntural; un tema de políticas públicas y no solo de asuntos administrativos o funcionales.

Si asumimos que los conceptos de salud y de derecho fundamental a la salud también parten de una visión integradora del sistema de fuentes de derecho, se

Jaime León Gañán Echavarría, Los muertos de Ley ioo.

49 Precisamente en el marco de los Objetivos de Desarrollo del Milenio se muestra que sin logros significativos en la reducción de la pobreza, la seguridad alimentaria, la educación, el empoderamiento de las mujeres y la mejora de las condiciones de vida en los barrios pobres, muchos países no alcanzarán las metas de salud.

Cfr. Toni Barbarà, “Toni Barbarà: sobre determinantes de la salud”, Punts de Vista (blog), 23 de mayo de 2008 , https://goo.gl/xsF648

Cfr. Alfonso Miranda Talero, El derecho de la Seguridad Social, ${ }^{\mathrm{a}}$ ed. (Bogotá: Fundación Cultural Javeriana, 1995), 290.

Por ello son de trascendental importancia las acciones encaminadas a fortalecer los derechos a la salud, a la educación y a la vivienda, a través de estrategias de Escuela Saludable y Vivienda Saludable con base en los mecanismos integrados en los "Lineamientos Nacionales de para la Aplicación y el Desarrollo de las Estrategias de Entornos Saludables" de los Ministerios de Protección Social, Educación Nacional, de Ambiente, Vivienda y Desarrollo Territorial de Colombia y la Organización Panamericana de la Salud de 2006. les, Observación General n. ${ }^{\circ}$ I4", Comité del Pacto de Derechos Económicos, Sociales y Culturales, consultado en septiembre de 2016 , https://goo.gl/odtKPe 
debe tener en cuenta cada una de las fuentes al momento de hacer efectivo el goce del derecho fundamental al trabajo y el de la salud, en procura de tan caros principios como el pro homines4, la dignidad humana y la igualdad real; y en ese mismo sentido, que los derechos y deberes constitucionales se articulen en forma sistémica hacia la consecución de los fines esenciales de una vida digna y con calidad, en especial bajo la fuerza normativa, jurisprudencial y doctrinal del llamado Bloque de Constitucionalidad ${ }^{55}$ que hoy, de manera categórica, coloca al derecho a la salud con una especial fuerza normativa y lo afora con acciones y mecanismos especiales de garantía, protección y de defensa; entonces, el sistema de IVC también debe responder a los requerimientos de su garantía material y de su justiciabilidad vía acciones y mecanismos de protección constitucional, legal y, por supuesto, de la garantía de participación de todas las personas.

En este marco conceptual, el sistema de ivc creado por la Ley i 22 de 2007 debe mutar hacia un sistema articulado bajo los parámetros de la Ley I $75 \mathrm{I}$ de 20I 5. Para tal efecto, un sistema integrado de ivc estaría inmerso en el esquema y aplicación práctica del Ciclo phva que implica: planear, desde políticas públicas para la salud, objetivos, metas y acciones a largo y mediano plazo de logros de salud con indicadores claros y concretos; ejecutar y desarrollar estrategias confiables de medición de las metas trazadas, verificar las acciones, los logros, los indicadores y resultados obtenidos; actuar sobre los resultados y la verificación realizada con medidas de control eficaces y mecanismos de corrección oportunos y pertinentes, para luego replantear la planeación y ajustarla a nuevas metas y logros. Este ciclo podría estar basado en el actual enfoque de la Supersalud que fortalece la supervisión por cumplimiento mediante la implementación de la supervisión basada en riesgos ${ }^{56}$.

Lógicamente, para realizar la anterior tarea será necesario un análisis de la normativa vigente, con el fin de eliminar las superposiciones de facultades entre diferentes entidades y simplificar las normas y reglas al respecto; previo análisis de las posibilidades reales de descentralización de tales potestades. También será necesario continuar con las acciones de promoción y prevención de conductas que atenten contra el Derecho Fundamental a la Salud y de su dimensión prestacional como servicio público esencial, en forma integrada y concurrente con los organismos facultados para ello.

54 Principio hermenéutico que implica la interpretación normativa que le sea más favorable a la persona humana y la aplicación de la norma más beneficiosa en relación con sus derechos humanos.

55 Mónica Arango Olaya, "El bloque de constitucionalidad en la jurisprudencia de la Corte Constitucional colombiana", Revista furídica Precedente, (2004): 79

56 Antonio Giuffrida, Daniel Andrés Pinzón Fonseca y César Augusto Piñeros García, "Supervisión basada en riesgos, la nueva apuesta de Supersalud", Revista Monitor Estratégico, núm.7 (enero-junio de 201 5): 8-i 6. https://goo.gl/7q4oIw 
Asimismo, es necesario fortalecer la capacidad de reacción, de respuesta, de información y de resolución ante las diferentes quejas y reclamos de la ciudadanía o de los actores involucrados. Quizás un plan de contingencia o de choque para descongestionar los procesos aún represados en la Supersalud sería conveniente. Una tarea pendiente en nuestro País sería la reglamentación y puesta en marcha de la figura del defensor de salud, que podría coadyuvar desde los intereses del paciente y de los diferentes actores de una forma, quizás más objetiva, imparcial y oportuna, siempre y cuando se le otorguen facultades, presupuesto, movilidad nacional y se fortalezcan los canales electrónicos de información en todo el territorio colombiano. A lo anterior se deben aunar políticas claras de autogobierno y gobierno transparente de todas las entidades; la concientización y capacitación permanente en derechos y deberes en salud, y por supuesto —en el caso de ser necesarias- sanciones ejemplificantes y efectivas. También sería importante que las entidades que ejercen la ivc fueran realmente independientes de sus vigilados y que sus funciones no se mezclaran entre sí. De la misma manera sería conveniente la estructuración real y efectiva de Redes de Controladores que pudieran ejercer tal función en forma articulada, coordinada y colaborativa.

Quizás sea necesario transformar el actual modelo del sGsss colombiano hacia un modelo de orientación más centrado en los principios, elementos y características del derecho fundamental a la salud, que en las teorías de la competencia regulada o del pluralismo estructurado. Quizás sea necesario optar por un modelo con mayor legitimación, con regulación y modulación fuerte desde el Estado, organismos de control articulados y descentralizados con gran capacidad logística, administrativa, funcional, financiera, con autonomía y con efectivas facultades sancionatorias; con real participación de las personas y de las entidades organizadas en pro de la ivc del Derecho Fundamental a la Salud y de un novísimo Sistema de Salud que podría estructurarse con participación de los agentes privados, públicos, solidarios o mixtos, en la prestación de los servicios de salud, pero sin la intermediación de agentes privados; o rediseñar las fuentes de financiamiento de la salud y de las formas de flujo de los dineros, entre otras posibles modificaciones. Tal vez, en este nuevo escenario, la Ivc pueda ser más oportuna y eficiente en bien de la comunidad, de la dignidad humana y, por supuesto, del bienestar y calidad de vida de todas las personas residentes en Colombia.

\section{Conclusiones}

Si bien, la tradicional concepción de ivc se ha encontrado ligada, en forma estrecha, a la dimensión de los servicios públicos — en nuestro caso al de la salud como un servicio público esencial-, también es cierto que con la iusfundamentalización de tal derecho hacia uno de tipo fundamental autónomo, tanto en lo 
individual como en lo colectivo, la noción de IVC también debería mutar hacia la garantía, el cumplimiento y el respeto de los contenidos y elementos esenciales del derecho como tal y, de igual forma, cumplir con su tradicional objetivo de hacer efectivos los indicadores de eficiencia, calidad, accesibilidad, aceptabilidad y disponibilidad como servicio público esencial.

La prolija regulación, la existencia de múltiples actores del sGsss y de regímenes excepcionados y especiales de salud, la superposición de facultades de IVC o el vacío de ellas, el complicado entramado de entidades que presenta el actual sistema de salud colombiano, la dispersión geográfica de los actores involucrados, el gran universo de entidades y organismos por vigilar, la falta de estructuras más dinámicas de IVc, hacen que en general, el proceso de IVC no sea tan eficiente, ni tan oportuno como se podría esperar y, por supuesto, que en la realidad no haya un verdadero y articulado sistema de IVc; no obstante los grandes esfuerzos realizados por las entidades encargadas de la IVc, en especial, de aquellas acciones implementadas por Supersalud para conjurar la creciente crisis del sGsss colombiano.

Si partimos de concebir la salud como un derecho seriamente fundamental y de la necesidad de un real sistema de ivc que procure una salud con calidad, con equidad e igualdad material para toda la población, entonces se debe propender por un nuevo modelo de salud y lógicamente por un sistema de IVc cercano a las personas, al territorio, con articulación de sus actores, con posibilidades reales de control y herramientas eficaces de gestión.

\section{Bibliografía}

Arango Olaya, Mónica. "El bloque de constitucionalidad en la jurisprudencia de la Corte Constitucional colombiana". Revista furídica Precedente (2004): 79. https://goo.gl/y8iavt.

Barbarà, Toni. Punts de Vista (blog). https://goo.gl/xsF648.

Comité del Pacto Internacional de Derechos Económicos, Sociales y Culturales. "Observaciones generales aprobadas por el Comité de Derechos Económicos, Sociales y Culturales”. Consultado en septiembre de 2016 . https://goo.gl/odtKPe.

Contraloría General de la República. "Funciones de la Contraloría General de la República". Consultado en septiembre de 20I6. https://goo.gl/tEtSpv.

Defensoría del Pueblo. "Defensorías delegadas. Defensoría Delegada para la Salud, la Seguridad Social y la Discapacidad”. Consultado en septiembre de 20r6. https://goo.gl/kWD8ko.

Dromi, Roberto. Derecho Administrativo, ro. ${ }^{a}$ ed. Buenos Aires: Ciudad Argentina, 2004

Gañán Echavarría, Jaime León. "De la Naturaleza Jurídica del Derecho a la Salud en Colombia”. Revista Monitor Estratégico, núm. 3 (enero-junio de 2013): 7-19. https://goo.gl/2atxJE.

Gañán Echavarría, Jaime León. Los muertos de Ley roo: Prevalencia de la libertad económica sobre el derecho fundamental a la salud. Una razón de su ineficacia. Caso del posC. Medellín: Universidad de Antioquia, 2013. 
Giuffrida, Antonio; Pinzón Fonseca, Daniel Andrés y Piñeros García, César Augusto. "Supervisión basada en riesgos, la nueva apuesta de Supersalud”. Revista Monitor Estratégico, núm.7 (enero-junio de 2015): 8-r6. https://goo.gl/7q4oIw.

Guerrero, Ramiro; Prada, Sergio. I.; Chernichovsky, Dov y Urriago, Juan. "La doble descentralización en el sector salud: Evaluación y alternativas de política pública”. Borrador de Informe Final, Concurso de Investigación Fondo Germán Botero de los Ríos, ProEsA, Universidad Icesi, octubre de $20 \mathrm{I}_{3}$. https://goo.gl/feggLH.

Miranda Talero, Alfonso. El derecho de la Seguridad Social, I ${ }^{\text {a }}$ ed. Bogotá: Fundación Cultural Javeriana, I995.

Organización de los Estados Americanos (ozA). Departamento de Derecho Internacional. "Protocolo adicional a la Convención Americana sobre Derechos Humanos en materia de derechos económicos, sociales y culturales 'protocolo de san salvador'". Consultado en septiembre de 20r6. https://goo.gl/vTjlya.

Organización Mundial de la Salud (oms). "Constitución de la Organización Mundial de la Salud".

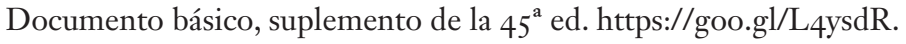

Pars, "Diagnóstico del Actual Modelo de Gestión de Inspección, Vigilancia y Control”. En Proyecto Evaluación y Reestructuración de los Procesos, Estrategias y Organismos Públicos y Privados Encargados de Adelantar las Funciones de Vigilancia y Control del Sistema de Salud. Informe final, Bogotá, Ministerio de la Protección Social.

Procuraduría General de la Nación. "Objetivos y funciones”. Consultado en septiembre de 20 r6. https://goo.gl/MIoRvJ.

Superintendencia de Industria y Comercio (sIC). "Objetivos y funciones". Consultado en septiembre de 2016. https://goo.gl/hPNFCv. 